\title{
Optimal Dispatch of Volunteers to Out-of-hospital Cardiac Arrest Patients
}

\author{
Niki Matinrad \\ Linköping University \\ niki.matinrad@liu.se \\ Nicklas Ennab Vogel \\ Linköping University \\ nicklas.ennab.vogel@liu.se
}

\author{
Tobias Andersson Granberg \\ Linköping University \\ tobias.andersson.granberg@liu.se \\ Vangelis Angelakis \\ Linköping University \\ vangelis.angelakis@liu.se
}

\begin{abstract}
Initiatives with mobile phone dispatched volunteers to out-of-hospital cardiac arrest (OHCA) cases, can be found today in some countries, e.g. Sweden, the Netherlands, Switzerland and Italy. When an OHCA case is reported, an alarm is sent to the registered volunteers' phones. However, the allocation of which volunteers to send to the automatic external defibrillator (AED) and who to send directly to the patient, is today based on simple rules of thumb. In this paper, we propose a model to optimally select how many and which volunteers to send directly to the patient, and who should pick up and deliver an AED. The results show that the model can help increase the survivability of the patients, compared to simple decision rules.
\end{abstract}

\section{Introduction}

Emergency services play a vital role in society as entities responsible for providing help to affected people and minimizing damages to public and private assets as well as the environment during emergencies. Existing resources that emergency services can utilize for their operations are limited, which creates a challenge. Besides resource shortage and cutbacks, emergency organizations also face the issue of long distances to sparsely populated areas [18]. At the same time, these organizations face societal changes, such as growing population and changes in demographic structures, as well as an increase in the number of people affected by larger events (disasters), increasing the need for emergency services.

One way of meeting these challenges is the increased utilization of volunteers [22], and one particular type of project that has been facing a rising interest in the past few years is mobile phone dispatched volunteers to out- of-hospital cardiac arrest (OHCA) cases [2, 3, 24, 25, 35]. In these projects, civilians who know how to do cardiopulmonary resuscitation (CPR) and to utilize automated external defibrillators (AED), register themselves. In case of an OHCA in their vicinity, they get a notification on their mobile phones, and if they can, they will respond to the call. A mobile phone positioning system (MPS) is used to locate the volunteers within a determined distance from the suspected OHCA patient, and a notification is automatically generated and sent to those volunteers; this is being done while emergency medical service (EMS) is being simultaneously dispatched to the patient [24, 25].

Although the notification and dispatching system utilizes MPS, depending on the situation, it may not be trivial to decide which and how many volunteers to dispatch, or which volunteers should pick up an AED before going to the patient, and who should go directly to the patient. Therefore, in this paper, we investigate whether applying optimization modelling for dispatch of volunteers can improve an OHCA patient's survivability. Hence, we present an optimization model to determine how the available volunteers should be dispatched to a suspected cardiac arrest case and compare the results from the proposed model with two simple, greedy dispatching approaches.

The remaining of the paper is organized as follows. First, in the following section, a review of the related literature is presented. The problem description and the optimization model can be found in Section 3. Section 4 is dedicated to the presentation of the solution procedure, while Section 5 contains the computational results, including the case description and input data. Finally, Section 6 concludes the paper and proposes future research. 


\section{Study baseline and related work}

For best possible utilization of volunteers, it is important to know their capabilities. One way to do this is to evaluate volunteers who have previously participated in response operations and describe and evaluate their characteristics [11]. The volunteers also need relevant training [30] and equipment [22]. Volunteer management systems can help supporting efficient utilization of this resource [28], as well as supporting coordination and information sharing [14]. An important input into these systems is then the previously mentioned capabilities, which have to be collected and registered [26].

Eventually, related tasks should be assigned to volunteers. In this regard, several qualitative studies have focused on task assignment, introducing concepts as crowdtasking and crowdsourcing of volunteers in emergencies and disasters $[1,12,17,20]$. In contrast to qualitative studies, one of the few quantitative studies in the field of volunteer management is [6] which based on a set of principals from the volunteer management field, proposes a multi-criteria optimization model for task assignment to both individual volunteers as well as volunteer groups.

One emergency where volunteers are used, is OHCA, where several studies indicate that quick help, will increase the patient survival rate (e.g. [4, 13, 32, 33]). Trials with lay persons (volunteers) show that mobile dispatch of volunteers shortens the time to first response [25], and publicly accessible AEDs might double the survival chance for the victims [10]. In [2], the volunteers are even only trained in using the AED, and thus not performing CPR.

When designing a volunteer initiative aimed at OHCA cases, it is important to be able to evaluate the effect, which most often is measured by the chance for survival. This can be modelled using a survival function, and to determine a suitable one, factors affecting patient's survivability should be found. These include time from collapse to CPR, from collapse to first defibrillator shock, from collapse to initiation of advanced cardiac life support [16], initial arrhythmia and the patient's age [13]. Relevant survival functions have been developed e.g. in [5, 31, 32, 33].

Comparing the work that is presented in this study with previous works, the following points are noted:

- There are not many quantitative studies in the volunteer management area. [6] is the quantitative work most relevant to this study considering task assignment to volunteers. While [6] is concerned with task allocation decisions and has no considerations of time in the model, the proposed model handles dispatching decisions for which time holds critical importance.

- In recent years, there has been an increase in studies utilizing volunteers in OHCA cases, especially from a practical perspective. As far as we know, there has not been any previous attempt to optimize the dispatching process, using mathematical modelling.

- There exist many studies of which factors that contribute to OHCA patient's survival, and how these can be combined into a survival function. Here we make an attempt of operationally using such a function when making dispatching decisions for volunteers.

\section{Problem description and mathematical model}

\subsection{Problem statement and assumptions}

The Volunteer to OHCA patient Dispatch (VOD) problem can be stated as:

The problem is to select how many and which volunteers to send directly to an OHCA patient, and who should first pick up an AED (and which AED), to maximize the patient's chance of survival.

The following assumptions are made:

- The set of volunteers and their locations are known.

- The set of AEDs and their locations are known, and they can be reached any time of the day. The time to retrieve an AED is negligible.

- Only one AED will be delivered to the patient.

- Some factors that in reality can be uncertain/stochastic are assumed to be deterministic such as all volunteers are available and will not decline an alarm, and that all travel times are predetermined and fixed.

- The arrival time of professional EMS is known and deterministic.

- All volunteers can perform CPR effectively for a fixed time period, $\tau$ minutes. After that, they need to rest for $r$ minutes, before they can perform CPR with full efficacy again. If no other volunteer can take over the CPR when the $\tau$ minutes has passed, there will be a gap, where the first volunteer continues with the CPR, but with decreased effectiveness. These gaps are penalized in the objective function.

Figures 1, 2 and 3 illustrate some intricacies of the volunteer CPR efficacy gap. Blue lines indicate the time that one of the volunteers is performing the CPR for $\tau=$ 2 minutes, and the red line is the $r=2$ minutes rest time after performing CPR with full effect (in Figure 1 and 2; 
in Figure 3, $r=3$ minutes). The green line in Figure 1 shows the time from the arrival of the second volunteer (v2) till he/she starts performing CPR as the first volunteer (v1) rests. As can be seen in Figure 1, from the start, CPR is performed continuously, without any efficacy gap by two volunteers until the arrival of EMS after 10 minutes. In Figure 2, the second volunteer arrives after the full effect period has passed, giving an "arrival gap", as indicated by the yellow column. Another type of gap, a "CPR gap", can be created when the rest time $(r)$ is longer than CPR performance time $(\tau)$ and not more than two volunteers are available. For instance, assume that $r=3$ minutes and $\tau=2$ minutes, as in Figure 3. Then, when volunteer 2 finishes performing CPR, volunteer 1 is still resting, which means that there will be a gap in providing effective care. Both arrival gaps and CPR gaps are penalized by the model.

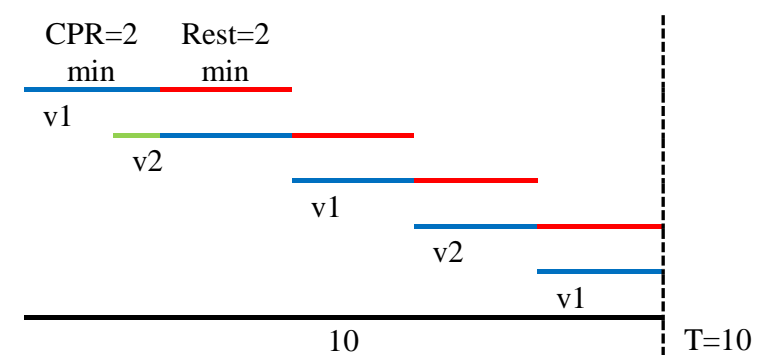

Figure 1. Sequence of performing CPR until the arrival of EMS, without any gap

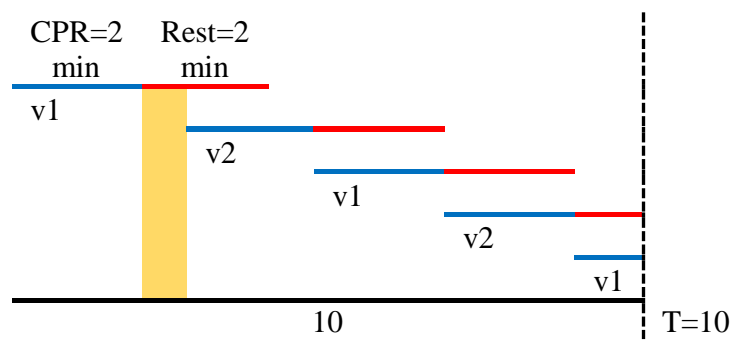

Figure 2. Sequence of performing CPR until the arrival of EMS, with arrival gap

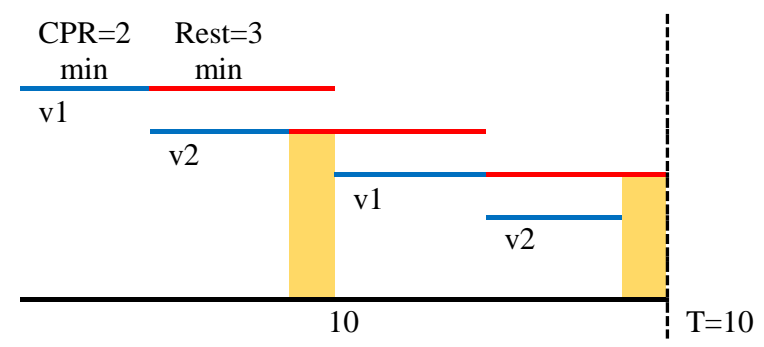

Figure 3. Sequence of performing CPR until the arrival of EMS, with CPR gap

\subsection{Model formulation}

The following notation is used when formulating the VOD problem as a mixed integer programming (MIP) model:

Sets/indices

I Set of volunteers indexed by $i \in I$

$L \quad$ Set of defibrillators indexed by $l \in L$

\section{Parameters}

$T$ Time horizon; i.e. arrival time of the EMS

$\tau \quad$ Volunteer CPR endurance time

$r \quad$ Volunteer rest time

$t_{i}^{p} \quad$ Travel time of volunteer $i$ directly to the patient

$t_{i l}^{D} \quad$ Travel time of volunteer $i$ to the patient with defibrillator $l$

$M \quad$ A large number

\section{Variables}

$x_{i}^{p} \quad 1$ if volunteer $i$ is assigned to the patient; 0 otherwise

$x_{i l}^{D} \quad 1$ if volunteer $i$ is assigned to getting defibrillator $l$ to the patient; 0 otherwise

$t^{*} \quad$ Time until arrival of first response, i.e. arrival of the first volunteer

$s^{*} \quad$ Time until start of defibrillation

$a_{i} \quad$ Arrival time of volunteer $i$ to the patient

$v_{i} \quad 1$ if volunteer $i$ is the first to arrive; 0 otherwise

$w_{i} \quad 1$ if volunteer $i$ is the last to arrive; 0 otherwise

$z_{i j} \quad 1$ if volunteer $j$ arrives directly after $i ; 0$ otherwise

$q_{1}$ Arrival gap; the possible gap in $\mathrm{CPR} /$ defibrillation between first volunteer on the scene and the following one, based on their arrival time

$q_{2} \quad$ CPR gap; the possible gap when the rest time $(r)$ is greater than endurance time $(\tau)$, and there are not enough volunteers available, or when there is only one volunteer available

It should be noted that by definition, $t^{*}$ is the time until arrival of first response. This might be either a volunteer dispatched directly to the patient, or a volunteer that arrives with an AED (in which case $t^{*}=$ $\left.s^{*}\right)$.

\section{Objective function}

The first objective function of the model aims to maximize the survivability of the OHCA patients. Thus, a survivability function (1) is developed, based on the time until arrival of first response and the time until start of defibrillation. Furthermore, an arrival or a CPR gap might affect the patient's survivability, so these should be avoided or minimized, giving rise to objective (2). 


$$
\begin{aligned}
& \operatorname{Max} \frac{1}{1+e^{\left(-1.3614+0.3429 t^{*}+0.18633 s^{*}\right)}} \\
& \operatorname{Min}\left(q_{1}+q_{2}\right) \\
& \operatorname{Min} \sum_{i}\left(x_{i}^{p}+\sum_{l} x_{i l}^{D}\right) \\
& \operatorname{Min}\left(-1.3614+0.3429 t^{*}+0.18633 s^{*}\right)
\end{aligned}
$$

The survival function (1) is maximized; this function is primarily based on the simplified logistic regression model presented in [31]. As the survival rate for OHCA patients in Sweden has increased by more than double between the years of 1992 and 2011 [29], to adjust the parameter values of the simplified logistic survival function and to reflect Swedish conditions for the year 2018 , the more comprehensive and representative study population data in [33] was used for the process of updating the survival function parameters.

The second function (2) is the sum of all gaps, which is minimized. As there does not exist enough studies (to the best of our knowledge) about the effect of gaps in delivering CPR on the survivability, it is not possible to directly incorporate this factor in the survivability function, although this would have been preferable.

While the presence of more than one volunteer is desirable, dispatching a high number of volunteers to one case potentially has two flaws: (1) they can get in the way of each other and hinder the EMS, and (2) if there is another case shortly after the first one, there might not be enough volunteers to respond to the second case. Consequently, the model aims to minimize the total number of dispatched volunteers, which is achieved through objective function (3).

The survival function (1), is nonlinear. This term is a logistic regression function, which is a nonlinear transformation of a linear regression function to produce numbers between 0 and 1 [7, 15]. By reverse transformation, i.e. utilization of $\log$ transform, the linear form can be obtained. Thus, maximizing (1) is equivalent to minimizing the power function in the denominator, resulting in Function (4).

\section{Constraints}

The constraints of the model are needed to ensure that enough, but not too many, volunteers are dispatched, to ensure the best sequence of volunteers' arrivals and handle possible gaps.

$$
a_{i}=t_{i}^{p} x_{i}^{p}+\sum_{l} t_{i l}^{D} x_{i l}^{D} \quad \forall i \in I
$$

$$
\begin{aligned}
& x_{i}^{p}+\sum_{l} x_{i l}^{D} \leq 1 \quad \forall i \in I \\
& \sum_{i} \sum_{l} x_{i l}^{D} \leq 1 \\
& t_{i}^{p} x_{i}^{p}+\sum_{l} t_{i l}^{D} x_{i l}^{D} \leq \mathrm{T} \quad \forall i \in I \\
& v_{i} \leq x_{i}^{p}+\sum_{l} x_{i l}^{D} \forall i \in I \\
& \sum_{i} v_{i} \leq 1 \\
& w_{i} \leq x_{i}^{p}+\sum_{l} x_{i l}^{D} \quad \forall i \in I \\
& \sum_{i} w_{i} \leq 1 \\
& t^{*} \geq a_{i}-M\left(1-v_{i}\right) \forall i \in I \\
& t^{*} \geq T\left(1-\sum_{i} v_{i}\right) \\
& s^{*} \geq \sum_{l} t_{i l}^{D} x_{i l}^{D} \quad \forall i \in I \\
& s^{*} \geq T\left(1-\sum_{i} \sum_{l} x_{i l}^{D}\right) \\
& \sum_{i} z_{i j} \leq 1 \forall j \in I \\
& \sum_{j} z_{i j} \leq x_{i}^{p}+\sum_{l} x_{i l}^{D} \forall i \in I \\
& z_{i j}+z_{j i} \leq 1 \forall i \in I, j \in I \\
& z_{i i}=0 \quad \forall i \in I \\
& a_{i} \leq a_{j}+M\left(1-z_{i j}\right) \forall i \in I, j \in I \\
& w_{i}+1+\sum_{j \neq i} z_{i j} \\
& \geq x_{i}^{p}+\sum_{l} x_{i l}^{D} \\
& +\frac{\sum_{j \neq i} x_{j}^{p}+\sum_{j \neq i} \sum_{l} x_{j l}^{D}}{|I|} \quad \forall i \in I \\
& w_{j} \leq v_{j}+\sum_{i \neq j} z_{i j} \forall j \in I
\end{aligned}
$$




$$
\begin{aligned}
& q_{1} \geq a_{j}-a_{i}-\tau-M\left(2-v_{i}-\right. \\
& \left.z_{i j}\right) \forall i \in I, j \in I \\
& q_{2} \geq r-\tau-T\left(\sum_{i}\left(x_{i}^{p}+\sum_{l} x_{i l}^{D}\right)\right. \\
& -2) \\
& x_{i}^{p}, x_{i l}^{D}, v_{i}, w_{i}, z_{i j} \in\{0,1\}, \\
& t^{*}, s^{*}, a_{i}, q_{1}, q_{2} \geq 0 \quad \forall i \in I, j \in I, l \in L
\end{aligned}
$$

Constraint (5) calculates the arrival time of each volunteer who either goes directly to the patient or first picks up an AED and takes it to the patient. Constraint (6) ensures that a volunteer either goes directly to the patient, or first picks up an AED, when he/she is dispatched. The maximum number of AEDs that can be picked up for each case (here, one) is handled by Constraint (7). Constraint (8) limits dispatch of volunteers to those who can arrive before the ambulance. Constraints (9) and (11) make sure that the first and last volunteers on site also have been dispatched to the patient, while (10) and (12) ensure that only one volunteer can be first, respectively last, on site. Constraint (13) establishes the time for the first volunteer's arrival to the patient. If for any reason no volunteer is dispatched to the patient, the time for the first response is determined through Constraint (14) and will be equal to arrival time of the EMS. If a volunteer is dispatched to pick up an AED, this time is determined by Constraint (15); otherwise, it is calculated by Constraint (16). Constraints (17)-(23) determine the sequence of the dispatched volunteers' arrival to the patient; Constraint (17) ensures that at most one volunteer arrives directly before volunteer $j$, while Constraint (18) makes sure that no volunteer can arrive after $i$, if $i$ is not dispatched. Constraint (19) ensures that if two volunteers have the same arrival time, they are still ordered, one after the other, while Constraint (20) makes certain that no volunteer will arrive after him/herself. Constraint (21) allows $z_{i j}$ to take the value 1 only if volunteer $i$ arrives before $j$. In case two or more volunteers are dispatched, at least one volunteer $(j)$ should come directly after volunteer $i$ (unless $i$ is the last to arrive) and consequently $z_{i j}$ is forced to be one (Constraint 22). Eventually, Constraint (23) determines the order of volunteers' arrival. Constraint (24) is responsible for calculating the arrival gap value for first two volunteers on site, i.e. if volunteer $j$ arrives after the endurance time of volunteer $i$ (when volunteer $i$ is first volunteer on site), $q_{1}$ is set to a positive value; otherwise it takes no value. Constraint (25) determines the value of the CPR gap, which may occur if the rest time is longer than the endurance time, and there are not enough volunteers available. It should be noted that Constraint (25) penalizes any dispatch of less than two volunteers regardless of $r$ and $\tau$. Thus, one volunteer carrying an AED will also be penalized since it is desirable to have at least two volunteers available at the scene, so that if for any reason, manual CPR is required, it is possible to perform this effectively. Constraint (26) is the set of binary and nonnegativity constraints for the decision variables.

\section{Solution procedure}

There are several different methods available for solving multi-objective optimization models. Some of these models are based on distance functions including goal programming, compromise programming, and the reference point method [27]. Some of the other methods scalarize multi objectives into a single objective and solve the problem, such as the weighted sum method and the $\varepsilon$-constraint method [23]. In this paper, the weighted sum method $[9,34]$ is adopted to solve the multi-objective, mixed integer optimization problem. In the proposed model, the three objectives Function 4, Function 2 and Function 3 are assumed to be $O b j_{1}$, $O b j_{2}$, and $O b j_{3}$. The single objective function is formulated as follows:

$$
\operatorname{Min} W_{1} O b j_{1}+W_{2} O b j_{2}+W_{3} O b j_{3}
$$

where $W_{1}+W_{2}+W_{3}=1, W_{1}, W_{2}, W_{3} \geq 0$ are the relative weights of the objectives in (27).

The model (objective function (27) and constraints (5)-(26)), was solved using AMPL and the solver CPLEX 12.7.1.0 on a computational server.

\section{Numerical experiments}

\subsection{Case description}

To test and validate the model, a case study is performed for the municipality of Norrköping, Sweden. The case is part of a more comprehensive research study, investigating the possibility of training and equipping people to be able to act as first responders as a new task within their current occupations (further described in [19]). An experiment was performed where a set of potential first responders were equipped with a smartphone, with an application installed, to which it was possible to send alarms. The responders could indicate in the application if they would accept the mission. The time of response and their location were noted. This made it possible to calculate their expected response time to the incident, using the GIS software ArcMap. As the alarms were based on historical data from the fire and rescue services, the old, real response times by the professional emergency services were 
Table 1. Characteristics of data from the volunteer project

\begin{tabular}{|c|c|c|c|c|c|c|c|}
\hline $\begin{array}{c}\text { number } \\
\text { of } \\
\text { scenarios }\end{array}$ & $\begin{array}{l}\text { Number of } \\
\text { volunteers }\end{array}$ & $\begin{array}{l}\text { Number } \\
\text { of AEDs }\end{array}$ & $\begin{array}{c}\text { Range of } \\
\text { travel time } \\
\text { to patient } \\
\text { [min] }\end{array}$ & $\begin{array}{c}\text { Range of } \\
\text { travel time to } \\
\text { AED } \\
\text { [min] }\end{array}$ & $\begin{array}{c}\text { Range of } \\
\text { EMS arrival } \\
\text { time } \\
{[\mathrm{min}]}\end{array}$ & $\begin{array}{c}\text { Volunteer's } \\
\text { endurance } \\
\text { time } \\
\text { [min] }\end{array}$ & $\begin{array}{c}\text { Volunteer's } \\
\text { rest time } \\
\text { [min] }\end{array}$ \\
\hline 174 & $1-7$ & 350 & $0.37-62.12$ & $0.48-213.1$ & $8.09-65.11$ & 2 & 2 \\
\hline
\end{tabular}

known. Locations of AEDs were collected from the Swedish AED registry [8] and expected travel times for the responders to AEDs and from AEDs to incidents were calculated using ArcMap.

During two months, a total of 149 alarms (i.e. 149 patients) were sent to the responders. In 141 cases, at least one responder indicated reception of the mission, and there existed historical travel times for the emergency services, making it possible to construct a scenario for the optimization model. However, the number of cases where at least two responders would have arrived before the emergency services (thus making the problem non-trivial), were only 33. So, in order to enlarge the dataset, the arrival times for the emergency services were extended, to be 3 minutes longer than the longest response time for any of the volunteer responders. This gave 141 additional scenarios, giving a total of 174 problem instances. Tables 1 presents the general characteristics of the scenarios. For all 174 instances, $r$ and $\tau$ are set to 2 minutes.

\subsection{Trade-off between objectives}

All three objectives are important, but their relative importance might be discussed. Starting with $\mathrm{Obj}_{3}$, while it is important not to dispatch more volunteers than necessary, we want the model to dispatch all volunteers that may contribute to an increased survival chance. We thus assume that there exists a cost for dispatching a volunteer, but that it is very low, and set $W_{3}$ to 0.01 .

Both $O b j_{1}$ and $\mathrm{Obj}_{2}$ are indicators for the survivability, but it is reasonable to assume that 1 minute prolonged time to CPR or defibrillation would be worse than a 1 minute gap. Thus, the weight $W_{l}$ should intuitively be larger than $W_{2}$. For an illustration of the trade-off between $\mathrm{Obj}_{1}$ and $\mathrm{Obj}_{2}$, consider the scenario in Table 2. There are four volunteers and two AEDs available. When the weights $W_{l}, W_{2}, W_{3}$ are set to $0.94,0.05,0.01$, the optimization model will dispatch Volunteer 2 and 3 directly to the patient while Volunteer 4 is dispatched to pick up AED 2. This results in an arrival gap of 1 minute, and an output of $23.9 \%$ from the survival function (1). Reducing $W_{l}$ to 0.84 , and increasing $W_{2}$ to 0.15 , Volunteer 2 and 4 are dispatched directly to the patient and Volunteer 1 is dispatched to pick up AED 1. In this solution there is no gap, and the survival function (1) output is $22.3 \%$. In this example, the survival chance for the patient as measured by (1), decreases slightly when the model prioritizes closing the gap. It is not obvious however, whether or not this decrease is acceptable, or if it would be better to have a gap in the effective delivery of CPR.

So, in order to investigate how the results in the 174 datasets are affected by varying $W_{l}$ and $W_{2}$ ( $W_{3}$ is always set to 0.01), different combinations are tested. Starting with $W_{l}=0.94$, the weight is reduced with intervals of 0.05 (while $W_{2}$ is increased). The main outcomes of this investigation show that:

- As expected, if there is no gap in arrival time when $O b j_{1}$ has its highest value (0.94), the model is insensitive to weight changes and the results for each of the objectives remains the same with the weight changes.

- If, on the other hand, there is a gap, the model is sensitive to weight changes. When there is a gap in the first output $\left(W_{1}=0.94\right)$, the first change in both $O b j_{1}$ and $O b j_{2}$ values, in $90 \%$ of cases, happens at $W_{1}=0.64$. After this point, there will be up to one more break point so that the gap reaches zero, but at what weight it occurs does not follow a pattern across the solved instances. The change in the value of $\mathrm{Obj}_{2}$ for the remaining $10 \%$ of the cases, always is to a zero gap in one step.

- All solutions produced by the model, where $W_{l}$ was less than 0.94 , can be considered dominated by the solution produced when $W_{l}=0.94$. This is because in order to close the gap, the model will dispatch volunteers with longer travel times, or select AEDs that are located further away from the patient, just to obtain arrival times for the

Table 2. Characteristic of designed scenario

\begin{tabular}{|c|c|c|c|c|}
\hline Volunteer & $\begin{array}{c}\text { Response } \\
\text { time } \\
\text { directly } \\
\text { to patient } \\
\text { [min] }\end{array}$ & \multicolumn{2}{|c|}{$\begin{array}{c}\text { Response time } \\
\text { including AED } \\
\text { pick up [min] }\end{array}$} & $\begin{array}{c}\text { EMS } \\
\text { arrival } \\
\text { time } \\
\text { [min] }\end{array}$ \\
\hline 1 & 7 & 8.5 & 19 & \\
\hline 2 & 3 & 13 & 12 & \multirow{2}{*}{14} \\
\hline 3 & 6 & 18 & 15 & \\
\hline 4 & 4 & 21 & 8 & \\
\hline
\end{tabular}


volunteers that are close to each other. Thus, for the tested scenarios, it was always best to have a high weight for $O b_{j}$, and no possible relevant trade-off between the objectives (like in the scenario in Table 2) was found.

- The model never dispatched more than two volunteers, which like in the previous bullet point, is likely due to the characteristics of the scenarios. If more volunteers were available, giving a larger possible solution space, it is likely that more volunteers also would have been utilized.

\subsection{Comparative results}

In the following results, a high weight (0.94) is allocated to the linearized survivability objective $\left(O b j_{1}\right)$, while $W_{2}$ is set to 0.04 and $W_{3}$ to 0.01 .

To investigate whether the proposed optimization model contributes to higher survivability of the patient or not, the results are compared to those produced by two simple, greedy dispatch approaches. These are inspired by dispatching approaches briefly mentioned in [35] and [20]. In both studies, dispatching is done by sending one third of volunteers directly to the patient and two thirds to the AED closest to the volunteers. However, neither of the papers clearly states how the task allocation is done, or the order of arrival of the volunteers. The two greedy approaches are presented in Table 3. In both greedy approaches, the limit of dispatched volunteers (i.e. $\left\lceil\frac{r}{\tau}\right\rceil+1$ ), showing total number of required volunteers, is based on the needed number of volunteers with endurance time $(\tau)$ to fill the whole rest time $(r)$ of the first volunteer $\left(\left\lceil\frac{r}{\tau}\right\rceil\right)$, plus one which indicates the first volunteer on site.

Figure 4 shows the objective function (27) values for the optimization model and the greedy approaches,

Table 3: Greedy dispatch approaches

\begin{tabular}{|c|c|c|c|}
\hline \\
\hline & Greedy 1 & & Greedy 2 \\
\hline 1 & $\begin{array}{l}\text { Select the closest volunteer and send her/him } \\
\text { directly to the patient, }\end{array}$ & 1 & $\begin{array}{l}\text { Select the volunteer who has the shortest response } \\
\text { time including the pickup of an AED, }\end{array}$ \\
\hline 2 & $\begin{array}{l}\text { Of the remaining of volunteers, select the one who } \\
\text { has the shortest response time including the pickup } \\
\text { of an AED, }\end{array}$ & 2 & $\begin{array}{l}\text { Considering the upper limit of dispatched volunteers } \\
\text { (i.e. }\left\lceil\frac{r}{\tau}\right\rceil+1 \text { ), dispatch the ones with the shortest } \\
\text { response time. }\end{array}$ \\
\hline 3 & $\begin{array}{l}\text { Considering the upper limit of dispatched } \\
\text { volunteers (i.e. }\left[\frac{r}{\tau}\right]+1 \text { ), dispatch the ones with the } \\
\text { shortest response time. }\end{array}$ & & \\
\hline
\end{tabular}

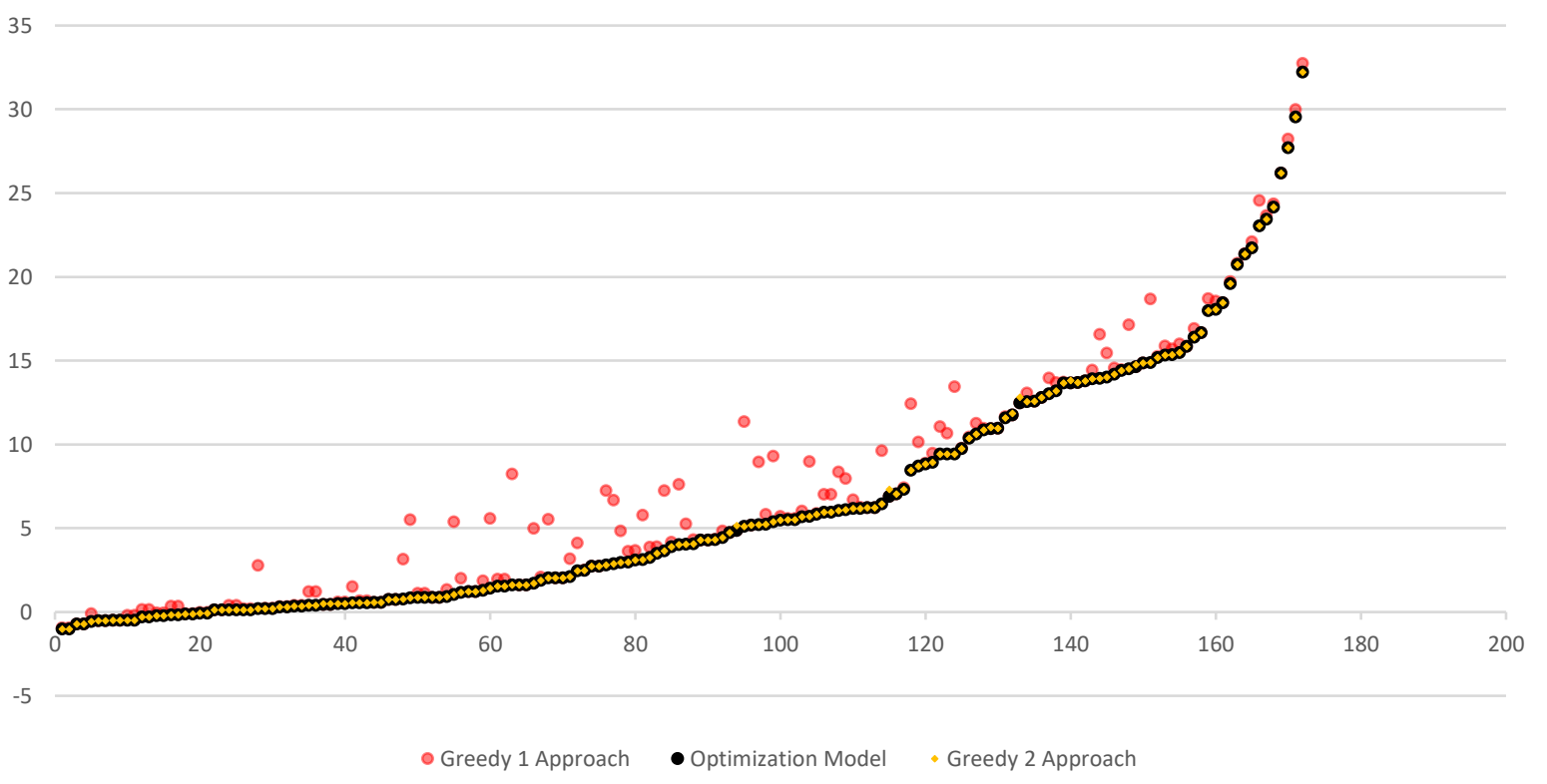

Figure 4. Weighted sum objective function values for optimization model and Greedy 1 and 2 approaches 
sorted in increasing objective function value for the optimal solutions.

The results show that for approximately $20 \%$ of the problem instances, both the optimization model and Greedy 1 produce the same dispatch decisions and hence, the objective function values and the patient's survivability chances are the same. This means that both optimization model and the Greedy 1 approach would send the first volunteer directly to the patient to perform CPR. However, for the remaining $80 \%$ of the problem instances, the optimization model has a lower (better) objective function value.

Greedy 2 behaves much more often like the optimization model. In only $7 \%$ of the 174 instances, Greedy 2 fails to produce an optimal solution. In the instances where the optimization model actually finds a better solution (i.e. when it is better to prioritize dispatch directly to the patient), the difference in objective function value is not very large. This is why it is difficult to discern the differences between the optimal results and Greedy 2 results in Figure 2. It should be noted though, that the solutions from Greedy 2 might have objective function (27) values up to $14 \%$ from optimal.

Table 4 presents one problem instance, in which neither the optimization model nor Greedy 1 result in any gap, and the response time of both dispatched volunteers are relatively short. However, the results indicate that even in such setting, the arrival of the first volunteer with an AED leads to better survivability for the patient.

The difference in survivability as estimated by (1) between the optimization model and Greedy 1 can be as large as $54 \%$ or as small as $0.00001 \%$. About $11 \%$ of these cases have more than $10 \%$ difference in survival probability, where the highest difference is produced when the optimization model results in $54.4 \%$ survival chance and the Greedy 1 approach in $0.8 \%$. In all of the instances where the optimization model produces a solution with a higher survival probability, a volunteer is dispatched to first pick up an AED, while Greedy 1 always dispatches the closest volunteer directly to the patient to perform CPR.
One of the problem types where Greedy 1 performs poorly compared to the optimization model is when there is only one volunteer available in the system. In these cases, the Greedy 1 approach will dispatch the volunteer directly to the patient, which means that the first possible defibrillation is when the EMS arrive. On the other hand, the optimization model will, in most cases, dispatch the volunteer to pick up an AED first. However, if the difference between the arrival of a volunteer with an AED and arrival of the EMS is not larger than 3 minutes, the volunteer will be sent directly to the patient by the model. This happens in $5 \%$ of the one-volunteer problem instances, resulting in the same solution by the Greedy 1 approach and the optimization model. Thus, the patient's survival probability is higher in the remaining $95 \%$ of the cases, when volunteers are dispatched according to the optimization model

Neither of the greedy approaches take into account the possibility of arrival gaps as a consequence of the dispatch decision, which can lead to lower survival chance for the patient. In about $30 \%$ of the instances, the Greedy 1 approach results in a larger gap compared to the optimization model and in all of these cases, the optimization model contributes to higher survival probability. This indicate that for about $30 \%$ of instances, the Greedy 1 approach not only contributes to less survival probability, but also produces a larger gap.

\section{Conclusion and future research}

As the resources that the emergency management system can use is limited, interest in less conventional types of resources such as volunteers is rising, and so is the attention drawn to projects utilizing volunteers in daily emergencies. For these to be successful, resource management systems that dynamically can handle volunteers as well as the traditional resources are essential. These systems, e.g. expert systems, require a reliable foundation, which can be supported by optimization modelling.

To investigate whether applying optimization modelling to dispatch volunteers can improve OHCA patients' survivability, we developed a multi-objective,

Table 4. Example of results from the optimization model and Greedy 1 approach without any gap

\begin{tabular}{|c|c|c|c|c|c|c|c|c|}
\hline \multirow[b]{2}{*}{ Model } & \multirow[b]{2}{*}{ Volunteer } & \multicolumn{2}{|c|}{ Dispatched } & \multirow{2}{*}{$\begin{array}{c}\text { Arrival } \\
\text { time to } \\
\text { patient } \\
\text { [min] }\end{array}$} & \multirow{2}{*}{$\begin{array}{c}\text { EMS } \\
\text { arrival } \\
\text { time } \\
{[\mathrm{min}]}\end{array}$} & \multirow[b]{2}{*}{ Obj 1} & \multirow[b]{2}{*}{$\mathbf{O b j}_{2}$} & \multirow[b]{2}{*}{$\begin{array}{c}\text { Survival } \\
\text { probability } \\
\text { (Function 1) }\end{array}$} \\
\hline & & $\begin{array}{l}\text { Directly to } \\
\text { the patient }\end{array}$ & $\begin{array}{c}\text { To pick up } \\
\text { an AED } \\
\text { first }\end{array}$ & & & & & \\
\hline \multirow{2}{*}{$\begin{array}{c}\text { Optimization } \\
\text { model }\end{array}$} & 1 & & $*$ & 4.26 & \multirow{4}{*}{8.91} & \multirow{2}{*}{0.89} & \multirow{2}{*}{0} & \multirow{2}{*}{0.2906} \\
\hline & 2 & $*$ & & 5.66 & & & & \\
\hline \multirow{2}{*}{$\begin{array}{l}\text { Greedy } 1 \\
\text { approach }\end{array}$} & 1 & $*$ & & 4.19 & & \multirow{2}{*}{1.17} & \multirow{2}{*}{0} & \multirow{2}{*}{0.2359} \\
\hline & 2 & & $*$ & 5.90 & & & & \\
\hline
\end{tabular}


mixed integer model and compared it to two greedy approaches. While the optimization model of course always produced the best solution, one of the greedy approaches, which prioritizes dispatch to pick up an AED, in a majority of the tested instances, also suggested an optimal dispatch. However, this may possibly be due to the instances tested, which were characterized by relatively few volunteers and many AEDs. If it would have been the other way around, it is possible that it would have been better to prioritize dispatch directly to patient instead. Now, the results indicate that the first volunteer should arrive with an AED; this happened in $80 \%$ of the instances. However, there are times (20\% of the problem instances) that dispatching a volunteer directly to the patient results in higher survivability. Therefore, as the dispatching decision has a direct effect on the life and death of OHCA patients, generalizing the dispatching decision to always prioritize the delivery of an AED might be too simplistic. Thus, it is useful to have an optimization model that can take the specific problem circumstances into account, and suggest an optimal dispatch decision, maximizing the survivability of the patient.

In reality, everything is prone to uncertainty. One of the future steps that can be taken is to consider this uncertainty for: the availability of volunteers when they get the notification (i.e. whether they will accept the mission or not), volunteers' travel times both directly to the patient and after picking up an AED, access time for an AED (i.e. the time after reaching the location of the AED, until travel towards the victim can start), and arrival time of the EMS. It should be noted that although there is an estimate for all these times, to depict reality better, they should not be treated as fixed which is why a stochastic approach might be suitable. All AEDs are considered available all the time in the presented model, but as some of these devices might not be available during the dispatch, their presence should also be treated as an uncertain element in the development of a stochastic model. In addition, in the current model, all volunteers are regarded as homogenous, while they might actually have varying capabilities, e.g. someone can perform CPR for longer time compared to another person, or might be a certified nurse, allowed to administer medicine. These differences can also be considered in a model but requires the systemization and collection of capabilities that are relevant for the specific task, in this case helping a person suffering from OHCA.

\section{References}

[1] D. Auferbauer, R. Ganhör, H. Tellioglu, and J. Pielorz, "Crowdtasking: Field Study on a Crowdsourcing
Solution for Practitioners in Crisis Management", in Proceedings of the ISCRAM 2016 Conference, 2016.

[2] A. Capucci, D. Aschieri, F. Guerra, V. Pelizzoni, S. Nani, G. Q. Villani, and G. H. Bardy, "Community-based automated external defibrillator only resuscitation for out-of-hospital cardiac arrest patients", Am Heart J, vol. 172, 2016, pp. 192-200.

[3] M. L. Caputo, S. Muschietti, R. Burkart, C. Benvenuti, G. Conte, F. Regoli, R. Mauri, C. Klersy, T. Moccetti, and A. Auricchio, "Lay persons alerted by mobile application system initiate earlier cardio-pulmonary resuscitation: A comparison with SMS-based system notification", Resuscitation, vol. 114, 2017, pp. 73-78.

[4] R. O. Cummins, J. P. Ornato, W. H. Thies, and P. E. Pepe, "Improving Survival from Sudden Cardiac-Arrest: The "Chain of Survival" Concept", Circulation, vol. 83, no. 5, 1991, pp. 1832-1847.

[5] V. J. De Maio, I. G. Stiell, G. A. Wells, and D. W. Spaite, "Optimal defibrillation response intervals for maximum out-of-hospital cardiac arrest survival rates," Annals of Emergency Medicine, vol. 42, no. 2, 2003, pp. 242-250.

[6] M. Falasca and C. Zobel, "An optimization model for volunteer assignments in humanitarian organizations", Socio-Economic Planning Sciences, vol. 46, no. 4, 2012, pp. 250-260.

[7] T. Fischetti, Data analysis with R, Packt, Birmingham, 2015.

[8] D. Fredman, M. Ringh, L. Svensson, J. Hollenberg, P. Nordberg, T. Djärv, I. Hasselqvist-Ax, H. Wagner, S. Forsberg, and A. Nord, "Experiences and outcome from the implementation of a national Swedish automated external defibrillator registry," Resuscitation, vol. 130, pp. 73-80, 2018.

[9] S. Gass and T. Saaty, "The computational algorithm for the parametric objective function", Naval Research Logistics (NRL), vol. 2, no. 1-2, 1955, pp. 39-45.

[10] P. W. Groeneveld and D. K. Owens, "Cost-effectiveness of training unselected laypersons in cardiopulmonary resuscitation and defibrillation," Am J Med, vol. 118, no. 1, 2005, pp. 58-67.

[11] W. J. Groh, A. Birnbaum, A. Barry, A. Anton, N. C. Mann, M. A. Peberdy, K. Vijayaraghavan, J. Powell, and V. N. Jr. Mosesso, "Characteristics of volunteers responding to emergencies in the Public Access Defibrillation Trial", Resuscitation, vol. 72, no. 2, 2007, pp. 193-9.

[12] D. Havlik, J. Pielorz, and A. Widera, "Interaction with citizens experiments: from context-aware alerting to crowdtasking", in ISCRAM, 2016.

[13] J. Herlitz, L. Ekstrom, B. Wennerblom, A. Axelsson, A. Bang, and S. Holmberg, "Effect of bystander initiated cardiopulmonary resuscitation on ventricular fibrillation and survival after witnessed cardiac arrest outside hospital", Br Heart J, vol. 72, no. 5, 1994, pp. 408-12. 
[14] P. T. Jaeger, B. Shneiderman, K. R. Fleischmann, J. Preece, Y. Qu, and P. Fei Wu, "Community response grids: E-government, social networks, and effective emergency management", Telecommunications Policy, vol. 31 , no. $10-11,2007$, pp. 592-604.

[15] G., James, D. Witten, T. Hastie, and R. Tibshirani, An introduction to statistical learning-with applications in R, Springer, New York, 2015.

[16] M. P. Larsen, M. S. Eisenberg, R. O. Cummins, and A. P. Hallstrom, "Predicting survival from out-of-hospital cardiac arrest: a graphic model," Annals of emergency medicine, vol. 22, no. 11, 1993, pp. 1652-1658.

[17] S. B. Liu, "Crisis Crowdsourcing Framework: Designing Strategic Configurations of Crowdsourcing for the Emergency Management Domain", Computer Supported Cooperative Work (CSCW), vol. 23, no. 4-6, 2014, pp. 389-443.

[18] K. Y. Mojir and S. Pilemalm, "Actor-centred emergency response systems: a framework for needs analysis and information systems development", International Journal of Emergency Management, vol. 12, no. 4, 2016, pp. 403434.

[19] K. Y. Mojir, S. Pilemalm, and T. Andersson Granberg, "Semi-professionals: emergency response as an additional task in current occupations," International Journal of Emergency Services, in press, 2018.

[20] G. Neubauer, A. Nowak, B. Jager, C. Kloyber, C. Flachberger, G. Foitik, and G. Schimak, "CrowdtaskingA New Concept for Volunteer Management in Disaster Relief", in International Symposium on Environmental Software Systems, 2013, pp. 345-356: Springer.

[21] R. W. Pijls, P. J. Nelemans, B. M. Rahel, and A. P. Gorgels, "A text message alert system for trained volunteers improves out-of-hospital cardiac arrest survival", Resuscitation, vol. 105, 2016, pp. 182-7.

[22] E. Ramsell, S. Pilemalm, and T. Andersson Granberg, "Using Volunteers for Emergency Response in Rural Areas: Network Collaboration Factors and IT support in the Case of Enhanced Neighbors", in The 14th International Conference on Information Systems for Crisis Response And Management, May 21-24, Albi, Occitanie Pyrénées-Méditerranée, France, 2017, vol. 14, pp. 985-995: ISCRAM Association.

[23] G.P. Rangaiah, Multi-objective optimization: techniques and applications in chemical engineering, World Scientific, Singapore, 2009.

[24] M. Ringh, D. Fredman, P. Nordberg, T. Stark, and J. Hollenberg, "Mobile phone technology identifies and recruits trained citizens to perform $\mathrm{CPR}$ on out-ofhospital cardiac arrest victims prior to ambulance arrival", Resuscitation, vol. 82, no. 12, 2011, pp. 1514-8.

[25] M. Ringh, M. Rosenqvist, J. Hollenberg, M. Jonsson, D. Fredman, P. Nordberg, H. Jarnbert-Pettersson, I. Hasselqvist-Ax, G. Riva, and L. Svensson, "Mobilephone dispatch of laypersons for CPR in out-of-hospital cardiac arrest", N Engl J Med, vol. 372, no. 24, 2015, pp. 2316-25.

[26] M. Romano, T. Onorati, P. Díaz, and I. Aedo, "Improving emergency response: Citizens performing actions", in ISCRAM, 2014.

[27] C. Romero, M. Tamiz, and D. Jones, "Goal programming, compromise programming and reference point method formulations: linkages and utility interpretations", Journal of the Operational Research Society, vol. 49, no. 9, 1998, pp. 986-991.

[28] J. Schönböck, M. Raab, J. Altmann, E. Kapsammer, A. Kusel, B. Pröll, W. Retschitzegger, and W. Schwinger, "A survey on volunteer management systems," in System Sciences (HICSS), 2016 49th Hawaii International Conference on, 2016, pp. 767-776: IEEE.

[29] A. Strömsöe, L. Svensson, Å. B. Axelsson, A. Claesson, K. E. Göransson, P. Nordberg, and J. Herlitz, "Improved outcome in Sweden after out-of-hospital cardiac arrest and possible association with improvements in every link in the chain of survival", European heart journal, vol. 36, no. 14,2014 , pp. 863-871.

[30] J. H. Sun and L. A. Wallis, "The emergency first aid responder system model: using community members to assist life-threatening emergencies in violent, developing areas of need", Emerg Med J, vol. 29, no. 8, 2012, pp. 673-8.

[31] T. D. Valenzuela, D. J. Roe, S. Cretin, D. W. Spaite, and M. P. Larsen, "Estimating effectiveness of cardiac arrest interventions: a logistic regression survival model," Circulation, vol. 96, no. 10, 1997, pp. 3308-13.

[32] T. D. Valenzuela, D. J. Roe, G. Nichol, L. L. Clark, D. W. Spaite, and R. G. Hardman, "Outcomes of rapid defibrillation by security officers after cardiac arrest in casinos", N Engl J Med, vol. 343, no. 17, 2000, pp. 12069.

[33] R. A. Waalewijn, R. de Vos, J. G. Tijssen, and R. W. Koster, "Survival models for out-of-hospital cardiopulmonary resuscitation from the perspectives of the bystander, the first responder, and the paramedic", Resuscitation, vol. 51, no. 2, 2001, pp. 113-122.

[34] L. Zadeh, "Optimality and non-scalar-valued performance criteria", IEEE transactions on Automatic Control, vol. 8, no. 1, 1963, pp. 59-60.

[35] J. A. Zijlstra, R. Stieglis, F. Riedijk, M. Smeekes, W. E. van der Worp, and R. W. Koster, "Local lay rescuers with AEDs, alerted by text messages, contribute to early defibrillation in a Dutch out-of-hospital cardiac arrest dispatch system," Resuscitation, vol. 85, no. 11, pp. 1444-9, Nov 2014. 